\title{
Subcutaneous and sublingual allergen-specific immunotherapy: a tale of two routes
}

\author{
${ }^{1}$ Cardiac/Pulmonary Rehabilitation, ASST Pini/CTO, Milan, Italy \\ ${ }^{2}$ Allergy Clinic, Casa di Cura Villa Montallegro, Genoa, Italy \\ ${ }^{3}$ Allergy and Clinical Immunology, Medicine and Surgery Department, University of Parma, Parma, Italy
}

\section{KeY wORDS \\ Allergen immunotherapy; respiratory allergy; subcutaneous route; sublingual route; \\ efficacy; safety.}

\author{
Corresponding author \\ Giorgio Ciprandi \\ Casa di Cura Villa Montallegro \\ Via Monte Zovetto 27 \\ 16132 Genoa, Italy \\ E-mail: gio.cip@libero.it
}

\section{Doi}

10.23822/EurAnnACI.1764-1489.150

\begin{abstract}
Summary
Specific immunotherapy is the only treatment acting on the causes and not only on symptoms of respiratory allergy. It was first introduced as subcutaneous immunotherapy (SCIT) with the aim to induce immunological tolerance to the administered allergen(s). In the 1980s, sublingual immunotherapy (SLIT) was developed, mainly to improve the safety, which was a critical issue at that time.

This article reviewed the available literature, including a large number of randomized controlled trials, meta-analyses, and real-life studies as well, on the outcomes of SCIT and SLIT concerning the treatment critical issues of the two routes, that are efficacy, safety, cost-effectiveness, and compliance to treatment.

The efficacy of SCIT and SLIT is similar in respiratory allergy, providing, based on the induction of typical changes in the immunologic response, an early control of symptoms that steadily increases during the treatment and its efficacy lasts after the recommended duration of three years. Such results are the reason why SCIT and SLIT have economic advantage over symptomatic drugs.
\end{abstract}

\section{Introduction}

Allergen immunotherapy (AIT) is defined as "the repeated administration of specific allergens to patients with IgE-mediated conditions for the purpose of providing protection against the allergic symptoms and inflammatory reactions associated with natural exposure to these allergens" (1). This outcome is obtained by the ability of AIT to induce a number of cellular and humoral effects that result from the shift from the Th2 lymphocyte dominated pattern, typical of the allergic response, to the Th1 pattern associated to tolerance, and from the generation of $\mathrm{T}$ regulatory cells $(2,3)$. As the fine mechanisms of AIT are being revealed, the need to identify reliable biomarkers predicting the response to treatment is an emerging issue $(4,5)$. The quality of allergen extracts used in AIT is crucial for inducing immunological changes and providing clinical success. This concerns both the initial injective route of administration, i.e. subcutaneous immunotherapy (SCIT) and the subsequently introduced sublingual immunotherapy (SLIT). The development of these two forms of AIT was quite different, because SCIT was initi- ated more than a century ago as a merely empirical treatment of hay fever (6), while SLIT was introduced only in the 1980s, when the knowledge on pathophysiology of allergy and mechanisms of AIT became clear (7). In this review, meta-analyses were preferred to evaluate the effectiveness and safety of SCIT and SLIT, while large-scale trials performed for registration from the regulatory agencies were used for the latest generation of SLIT products. Also real-life studies on very large patients population were considered.

\section{The milestones in the development of allergen immunotherapy}

The injective route was introduced in 1911 although the pathogenesis of allergic disease was not fully elucidated until the discovery of $\operatorname{IgE}$ antibodies in the 1960s (8), which clarified the mechanisms of allergy and the immunologic modifications achieved with AIT $(9,10)$. Despite this, safety issues emerged in the 1980s, when fatal reactions to SCIT from the UK (11) and USA (12) were described. Nowadays it has been clarified that 
the actual risk factor for fatal reactions to SCIT is the presence of uncontrolled asthma at the time of the allergen extract injection; in fact, fatalities become extremely rare when patients with uncontrolled asthma are excluded from this treatment (13). However, in the 1980 this factor was not yet acknowledged, thus the development of other routes of administration was deemed essential. Therefore, the oral route and the local nasal route were investigated, although these types of administration were reconsidered due to the high allergen doses required for efficacy and the repeated nasal reactions to the administered allergen, respectively (14). In fact, oral immunotherapy remains under investigation as a treatment for food allergy but non for respiratory allergy, while local nasal immunotherapy was abandoned. Indeed, the development of SCIT and SLIT, as summarized in table I, proceeded through stages of evolution showing similarities and differences. The current advance is the ongoing registration of immunotherapy products, based on the fulfillment of criteria from the International Conference on Harmonisation of Technical Requirements for Registration of Pharmaceuticals for Human Use. In fact, until recently all allergen extracts were classified as named patients products (NPP), that are defined by their production through at least one industrial process based on individual medical prescription, not responding to the above criteria. Some NPP were registered at local level, with no acknowledgement by other nations. Nowadays, guidelines for product registration from the European Medicines Agency are available regarding production and quality issues (15) and the adherence to such guidelines has already resulted in internationally registered products, which will be discussed below.

\section{Similarities of SCIT and SLIT}

\section{Efficacy of SCIT}

After decades of open studies on SCIT, the first placebo-controlled trials were commonly based on small populations of patients, often not higher than 30-35 subjects, about $50 \%$ of whom treated with placebo. Such low numbers obviously exposed the results to the statistical risk of stochastic observations. Similar problems concerned SLIT after its introduction. The risk of poor statistical power was managed by using meta-analysis, which has the crucial advantage to aggregate the information and thus to achieve a higher statistical power and a more solid point estimate than obtained from any individual study. Still, meta-analysis also has the limit to be biased by the methods of search and selection of studies of the investigator, incomplete data, and the kind of data analysis (16). An appropriate way to address this limit is to use the Cochrane approach, which was specifically designed to reduce investigator-related bias (17). Table II shows the main results from meta-analyses of randomized double-blind, placebo-controlled trials (DBPC) of SCIT
Table I - Stages of evolution of SCIT and SLIT.

\begin{tabular}{ccc}
\hline & SCIT & SLIT \\
\hline Birth & 1911 & 1986 \\
\hline First controlled trial & 1966 & 1986 \\
\hline $\begin{array}{c}\text { First meta-analysis } \\
\text { studies on large } \\
\text { populations }\end{array}$ & 1995 & 2003 \\
\hline $\begin{array}{c}\text { Products } \\
\text { registration }\end{array}$ & $\begin{array}{c}\text { Ongoing, thus } \\
\text { far registered } \\
\text { products only in } \\
\text { single nations }\end{array}$ & $\begin{array}{c}\text { International registration } \\
\text { for grass pollen and dust } \\
\text { mites tablets; } \\
\text { ongoing for ragweed } \\
\text { pollen } \\
\text { tablets }\end{array}$ \\
\hline
\end{tabular}

in patients with allergic asthma or allergic rhinitis (18-23). In particular, Abramson et al. performed from 1995 to 2010 three Cochrane meta-analysis on asthma. The latest meta-analysis update included 88 trials, 42 of them concerning SCIT with dust mites, 27 with pollens, 10 with animal dander, 2 with latex, 2 with molds, and 6 with multiple allergens, using the standardized mean difference (SMD) as analysis parameter (21). A significant improvement in asthma symptom scores was found (SMD - $0.59,95 \%$ CI -0.83 to -0.35 ) and it would have been necessary to treat 3 patients with SCIT to avoid one deterioration in asthma symptoms and to treat 4 patients to avoid one patient requiring increased drug treatment. SCIT significantly decreased both allergen specific and non-specific BHR (20). Based on the level of evidence, no further Cochrane meta-analysis was performed, while other meta-analyses addressed aspects such as efficacy in asthmatic patients treated with dust mite extracts (23), or the outcome of SCIT compared with pharmacotherapy (22). For the latter, besides the usual SMD, also by the relative clinical impact (RCI), which is the percentage reduction in TSSs and TNSSs obtained with active treatment compared with placebo was used, with a significantly better result for SCIT than for mometasone $(-31.7 \% \pm 16.7 \%, \mathrm{p}<0.00001)$ and montelukast $(6.3 \% \pm 3.0)$.

\section{Efficacy of SLIT}

The first meta-analysis on the efficacy of SLIT on allergic rhinitis was performed in 2015 on 22 randomized trials (24). A significantly higher efficacy of SLIT versus placebo was found, as assessed by an SMD of -0.42 for symptom scores $(\mathrm{p}=0.002)$ and -0.43 for medication scores $(\mathrm{p}=0.00003)$. Due to the relatively low numbers, the authors were not able to detect differences in patient subgroups defined by age and the kind of allergen, 
Table II - Efficacy of SCIT.

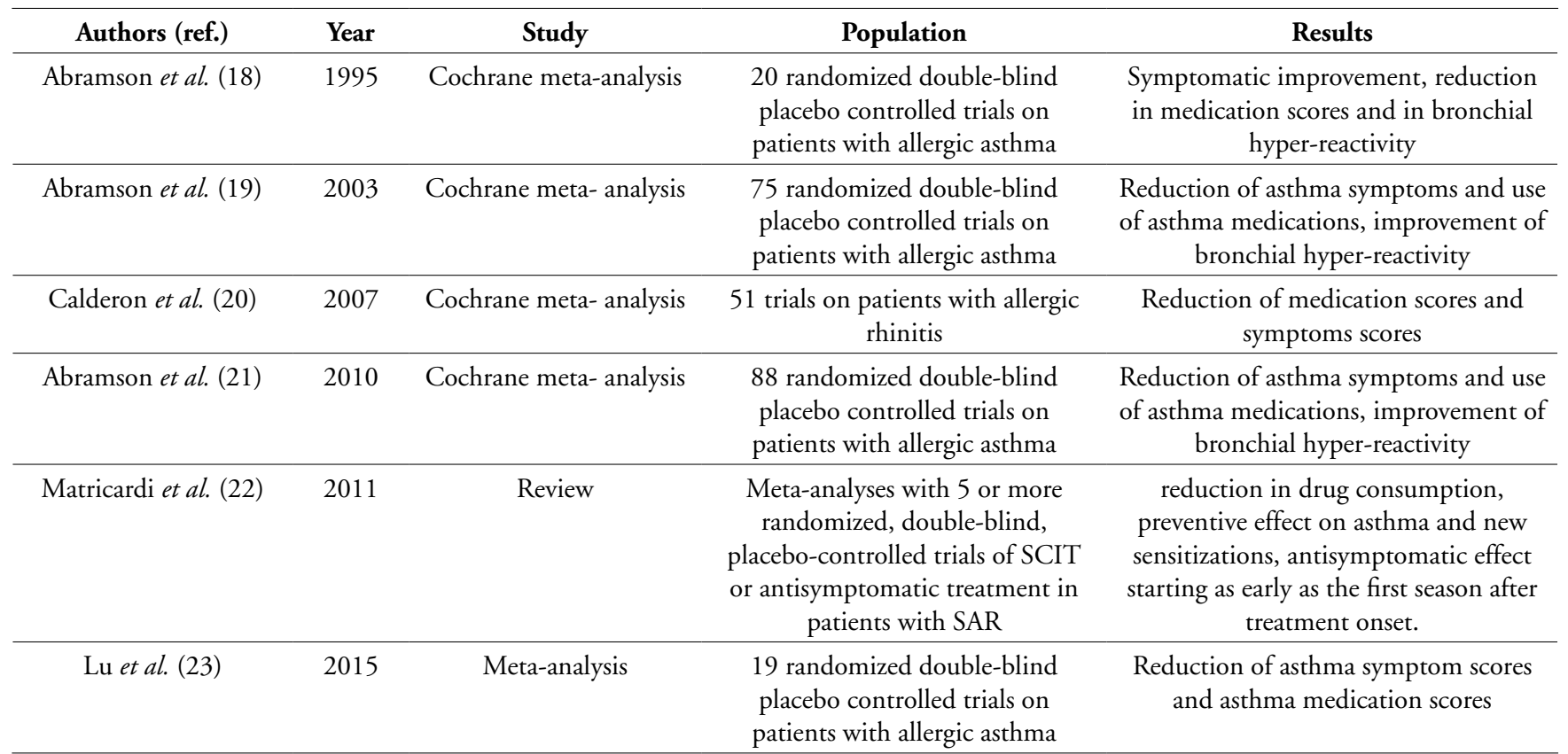

but this was possible in subsequent analyses. Table III reports the main results of further meta-analyses on allergic rhinitis or asthma (24-34). The most recent meta-analyses included both SCIT and SLIT trials. In particular, the meta-analysis endorsed by the European Academy of Allergy and Clinical Immunology, including 61 SCIT trials and 71 SLIT trials demonstrated significant reductions in AR symptom (SMD -0.53), medication (SMD -0.37) and combined symptom and medication scores (SMD -0.49) with active treatment, with no significant difference between SCIT and SLIT (32). The guidelines from the European Academy of Allergy and Clinical Immunology (EAA$\mathrm{CI}$ ), based on the level of evidence stated that in patients with AR both SCIT and SLIT can be recommended (35). However, in a meta-analysis comparing SCIT and SLIT in patients with allergic asthma SCIT improved quality of life and decreased allergen-specific airway hyperreactivity, while SLIT did not reach such outcome (33).

As hinted above, the need to fulfill the rigorous process of registration as pharmaceutical therapies required by regulatory agencies resulted in the performance of large trials on the new SLIT products in standardized tablets. The recent preparations for SLIT in tablets of grass pollen extract were the first to be assessed. A pre-requisite was the inclusion of patients' populations much larger than commonly used previously, to make unlikely casual observations and thus making meta-analysis unnecessary. Actually, the two trials on the 1-grass tablets and the 5-grass tablets included 855 and 628 adults, respectively $(36,37)$. In the trial on the 1-grass (Phleum pratense) tablets patients were randomized to receive sublingually $2500,25,000$, or $75,000 \mathrm{SQ}$ (Standard Quality)-T (the units used by the producer to measure allergen activity) or placebo once daily for a mean duration of 18 weeks. The average rhinoconjunctivitis scores during the grass pollen season were reduced by $16 \%$ and the medication use was reduced by $28 \%$ for the grass tablet $75,000(\mathrm{p}=0.047)$ compared with placebo. A significant improvement in rhinoconjunctivitis QoL scores $(\mathrm{p}=0.006)$ and in the number of well days $(\mathrm{p}=0.041)$ were observed (36). In the trial on 5 -grass tablets three dosages for once daily administration were tested, namely 100, 300 and 500 IT (Index of Reactivity) and compared with placebo. SLIT was started 4 months before the expected grass pollen season and continued throughout the season. The 300IR and 500-IR doses significantly reduced the mean rhinoconjunctivitis total symptom score $(3.58 \pm 3.0, \mathrm{p}=0.0001$ and 3.74 $\pm-3.1, \mathrm{p}=0.0006$, respectively) compared with placebo (37). The 5-grass tablets were also evaluated in 278 pediatric patients (aged 5-17 years) with rhino-conjunctivitis receiving 300-IR once-daily or placebo, with the same time horizon of the study in adults. The SLIT treated group showed a mean improvement in rhinoconjunctivitis total symptom score of $28 \%$ compared with placebo, this being significantly better $(\mathrm{p}=0.001)$. Significant differences between active and placebo treatment were also found concerning the rescue medication score and the propor- 
Table III - Efficacy of SLIT.

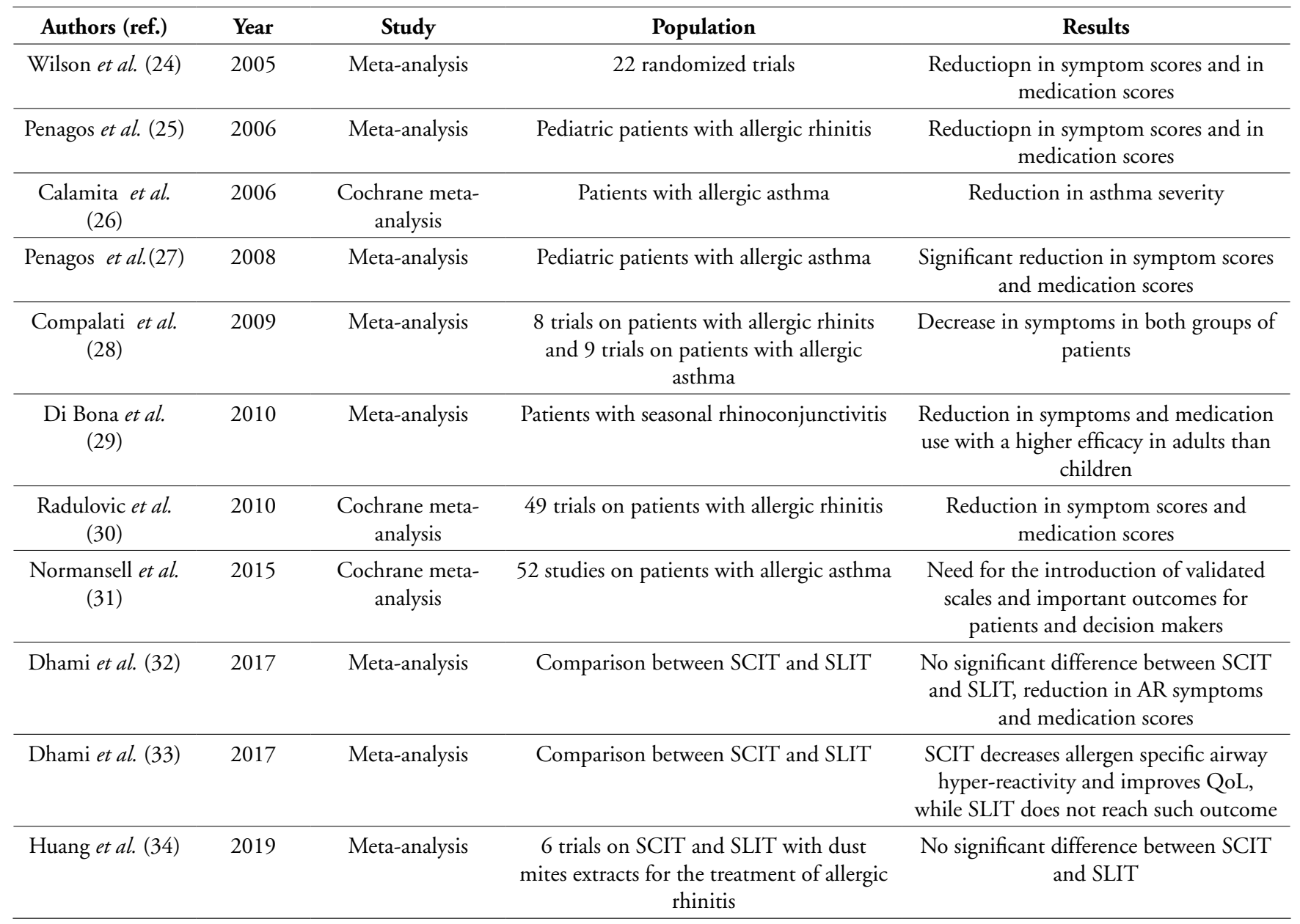

tion of days using rescue medication throughout the pollen season ( $\mathrm{p}=0.0064$ and $\mathrm{p}=0.0146$, respectively) (38). Both 1-grass tablets and 5 -grass tablets trials clearly demonstrated the need to administer high allergen doses to achieve clinical efficacy. Of interest, in a post-hoc analysis of data from the 5-grass tablets trials, Devillier et al. found that the magnitude of efficacy was higher in patients with more severe symptoms during the grass pollen season (39).

In 2014, the first trial on the efficacy of dust mite SLIT tablets was published. The trial included 604 patients (aged more than 14 years) with mite-induced rhinitis and mild-to-moderate asthma, who were randomized to receive 1,3 , or 6 SQ dust mite tablets or placebo. The primary endpoint was the use of inhaled corticosteroid (ICS), which was adjusted at baseline and the end of one year of treatment to the lowest dose giving asthma control. The 6 SQ dose reduced the daily ICS dose by a mean difference of $81 \mu \mathrm{g}$ compared with placebo $(\mathrm{p}=0.004)$.
The most common adverse events were local oral reactions, with a higher rate and severity for 3 and 6 SQ-dust mite than for 1 SQ-dust mite and placebo (40). This outcome resulted in the important goal to take account in the 2017 update of the Global Initiative on Asthma (GINA) guideline of dust mite tablets as add-on therapy in mite allergic adult patients who have asthma exacerbations despite ICS treatment, with a FEV1 value of at least $70 \%$ of predicted (41).

The dust mite tablets standardized in IR were evaluated instead as a treatment for mite-induced AR. From a group of 509 participants, patients were randomized to receive once daily 500 IR tablets, 300 IR tablets or placebo for one year, with a year of follow-up after the end of treatment. The two SLIT doses of 500 and 300 IR significantly reduced AR symptoms, as measured by the Average Adjusted Symptom Score (AASS), compared to placebo, by $20.2 \%(\mathrm{p}=0.0066)$ and $17.9 \%(\mathrm{p}=0.015)$, respectively. With both active treatments the efficacy was maintained 
during the one-year follow-up with no treatment (42). Further support for effectiveness of AIT was provided by real-life (also called real-world) studies. The value of these studies is to verify if the results obtained in controlled trials are reproduced in patients managed in routine clinical practice. In fact, the rigid inclusion criteria make unlikely the admission of such patients to trials, making uncertain their clinical response. The first paper mentioning the term real-life for AIT dates back to 2004 (43). Since then, more than 20 real-life studies on the effectiveness of SCIT and SLIT were published and recently reviewed (44). In the present review, only the studies on very large population of patients will be considered. In the study by Zielen $e t$ al. 2851 patients treated with grass pollen SLIT tablets and 71,275 control patients were analysed by a retrospective multiple regression analysis of data from a German prescription database over a period of 7 years. Changes over time in symptomatic drug consumption after SLIT withdrawal, use of medications for asthma, and time of asthma onset in patients with AR were used as indicators. A significant difference in favour of SLIT was detected for all comparators. In particular, the use of symptomatic drugs for AR compared to the pre-treatment period was $18.8 \%$ lower $(p<0.01)$ in SLIT treated patients than in controls, the asthma medication use decreased by an additional $16.7 \%(\mathrm{p}=0.004)$ after treatment withdrawal in SLIT treated compared with controls, and the onset of asthma was less frequent (odds ratio 0.696, $\mathrm{p}=0.002$ ) in SLIT treated patients than in controls (45). Another large real-life study used the same German prescription database and a time horizon of 2-6 years on a retrospective cohort of 9001 patients treated with SCIT or SLIT for birch pollen-induced AR and asthma and 45,005 patients treated only with symptomatic drugs as controls. AIT was performed by different birch or other Betulaceae (hazel, alder) pollen extracts, administered by natural pollen SCIT, allergoid preparations for SCIT and SLIT drops. At the end of a 6 years follow-up, the results of multiple-regression analysis showed that the rate of patients no longer taking symptomatic drugs for AR was $65.4 \%$ of AIT treated patients vs. $47.4 \%$ of controls $(\mathrm{p}<0.001)$. Concerning asthma, the rate of patients using no longer using anti-asthmatic drugs was $49.1 \%$ of AIT treated patients vs. $35.1 \%$ of controls $(\mathrm{p}<0.001)$. The evaluation of the risk of developing asthma in patients with only AR was significantly lower in AIT treated vs. controls (odds ratio $0.83, \mathrm{p}=0.001$ ) (46). The most recent real-life study analyzed the prescription fulfilment data collected from French retail pharmacies from 2012 to 2016 . Using linear regression analyses, 1099 patients who had received at least two prescriptions of grass pollen SLIT tablets for at least two consecutive years were compared with 27,475 control patients who had received only symptomatic medications. A 50\% decrease in the use of symptomatic AR medications was observed, compared with a $30 \%$ increase in the control group without age matching ( $p<0.0001$ vs. SLIT) and a $20 \%$ increase in the control group with age matching $(\mathrm{p}<0.0001$ vs. SLIT). Regarding asthma prevention, during the follow-up $1.8 \%$ of SLIT-treated patients and $5.3 \%$ of controls initiated asthma treatment. A lower risk of drug dispensing for new onset asthma was observed in SLIT treated $v$ s. controls, by $62.5 \%$ without age matching $(\mathrm{p}=0.0025)$ and by $63.7 \%$ with age matching $(\mathrm{p}=0.0018)$. The authors concluded that prescription of grass SLIT tablets lessens the dispensing of $\mathrm{AR}$ and asthma medications in real life (47).

\section{Immunological mechanisms of AIT}

The exclusive disease-modifying outcome of AIT is due to its immunological effects on IgE-mediated allergic diseases. Successful AIT can in fact restore immune tolerance to allergens, by the inhibition of the early and late allergic response, the induction of allergen-specific regulatory $\mathrm{T}$ (Treg) and $\mathrm{B}$ cells (Breg) and the increase in production of anti-inflammatory cytokines, such as IL-10 and TGF- $\beta(2,3)$. It is known that in allergic diseases the dis-regulation of Th2 response induces an increased release of cytokines such as IL-4, IL-5 (which stimulate the IgE class switch of B cell), and IL-13 (48). IgE exposed on the cellular membrane of mast cells and basophils bind the specific allergens, thus inducing the degranulation and the release of preformed mediators, such as histamine and leukotrienes, and generating the allergic response. IL-5 has a major effect on eosinophils by activating them and prolonging their survival rate, in addiction it stimulates the growth of B cell (49). These immunologic effects of AIT result in a long-lasting tolerance to the specific allergen, by decreasing the number and the activation of eosinophils and mast cells, and modulating the response and the activity of $\mathrm{T}$ and $\mathrm{B}$ cells, reducing the production and the release of IgE while increasing IgG4, a subclass of blocking antibody which compete with IgE for binding with the allergens, causing the reduction in activation of basophils and $\mathrm{B}$ cells. Although routes, doses of allergen and site of administration differ between SLIT and SCIT, the final effect in the modulation of the immune network is the same: the induction of peripheral tolerance mediated by Treg, which modulates the activation and the survival of peripheral immune cells by the release of anti-inflammatory cytokines such as IL-10 with suppression of $\operatorname{IgE}$ production and inhibition of $\mathrm{B}$ cells proliferation (50). Figure 1 summarizes the main mechanisms of action if SCIT and SLIT. They result in the persistence of the clinical effects after stopping the treatment and the preventive capacity to interfere with the natural history of $\mathrm{AR}$, and particularly the development of asthma. As to the first effect, a number of long-term studies, often following an initial trial, showed that treatment durations longer than three years resulted in prolonged maintenance of AR symptoms control over time (51). A recent review analyzed the evidence for long-term effects of SCIT and SLIT assessed 
through placebo-controlled, randomized clinical trials including a follow-up of at least 1 year after treatment withdrawal. The data suggest the evidence on the adequacy of 3 years of either SCIT or SLIT in providing allergen-specific tolerance maintained for at least 2-3 years after treatment stopping (52). This observation supports the need to avoid a duration shorter than 3 years.

In addition, there is good evidence on the ability of both SCIT and SLIT to prevent the progress from AR to asthma. Following initial investigation on small groups of patients, the preventive allergy treatment (PAT) study enrolled 183 children with grass and/or birch pollen allergy undergoing SCIT or drug treatment, assessing the development of asthma by clinical evaluation. After 3 years of SCIT, in the period of follow-up performed for up 5 years a significantly less frequent onset of asthma was found in comparison with drug-treated children (odds ratio 2.68 (1.3-5.7)) (53). Several other studies, also addressing SLIT, confirmed such outcome, as recently reviewed (54). Indeed, no such evidence was achieved for the AIT capacity to prevent new sensitizations. This modification of the evolution of atopy was initially suggested by Des Roches et al., who followed-up for 3 years a group of 22 children monosensitised to dust mites and treated with SCIT, comparing them to 22 matched non SCIT treated children. All 22 non-treated children developed new sensitivities $v s$. only 10/22 SCIT treated children $(\mathrm{p}<0.01)$, suggesting that AIT in children monosensitised to dust mites can prevent new sensitizations (55). However, this outcome is far from clear, as demonstrated by the contrasting results of subsequent studies (56). A recent meta-analysis on 18 studies including 1049 children and 1057 adults concluded that low evidence supports the ability of AIT to prevent the onset of new sensitizations, the highest benefit being reported in small studies with a shorter follow-up. Still, the authors commented that high quality trials could change this estimate (57).

Figure 1 - Mechanisms of action shared by SCIT and SLIT. Sublingual and subcutaneous immunotherapy. In SCIT the allergen contacts the antigen presenting cells (APC) of the skin while in SLIT APC involved are those in the sublingual mucosa. Both SCIT and SLIT induce immunotolerance. The induction of Treg results in early desensitization of mast cell and basophil and their suppression, together with eosinophils. IL-10 and TGF beta induce immunoglobulin class switch, with a decrease of IgE and induction of IgG4. Th2 response and cytokines result suppressed too. Inflammatory dendritic cell (DC) are suppressed in favor of tolerogenic DC.

SCIT
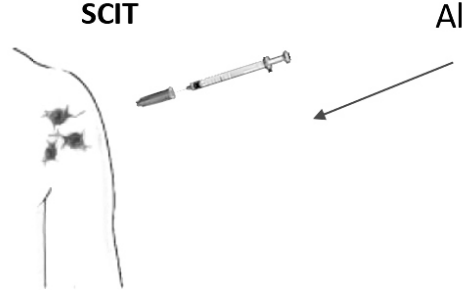

Allergen extract
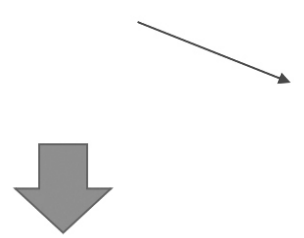

\section{SLIT}

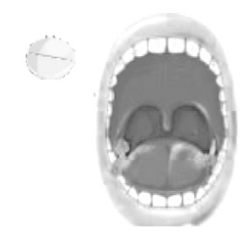

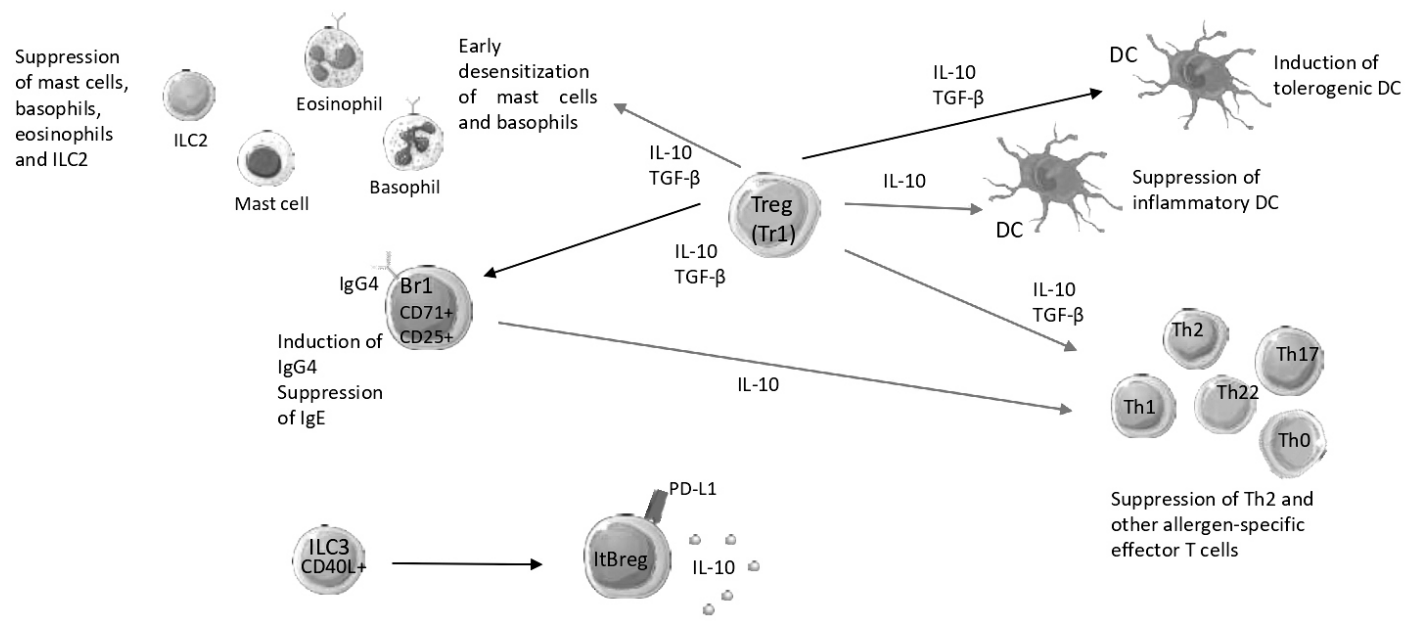




\section{Practical application of AIT}

The practice patterns of SCIT in Europe and US are significantly different, being mostly based on single allergens in Europe, while including an average of 8 allergens in US (58). Nelson analyzed 13 studies based on simultaneous administration of two or more unrelated allergens published from 1961 to 2007. Only few studies were well-designed and powered DBPC trials, and direct comparison between single-allergen and multiple-allergen was rarely performed. The author concluded that multiple-allergen can be effective but claimed for further investigation (59). Concerning SLIT, Calderon et al. studied the published evidence on the efficacy of the two strategies in polysensitized patients. According to the results of post-hoc analyses of DBPC trials, the efficacy of SLIT with grass-pollen tablets was found to be similarly effective in monosensitised and polysensitized patients. The authors also analyzed a number of studies from Europe evaluating SLIT in polysensitized patients using only the most clinically important allergen in two thirds of patients and a mix of two allergens in the remaining patients: a significant improvement in symptom and medication scores was achieved. Calderon $e t$ al. concluded that "multi-allergen immunotherapy in polysensitized patients needs more supporting data from large clinical trials to validate it as a treatment option" (60). A Workshop organized by the National Institute of Allergy and Infectious Diseases (NIAID) and the Agency for Healthcare Research and Quality (AHRQ) was aimed at developing trial concepts able to improve the use and effectiveness of AIT for respiratory allergy. One of the task to be addressed by the Expert groups is "to propose a study design to compare the effectiveness and safety of AIT by using 1 or a few allergens versus all or most allergens to which a patient is sensitized" (61). It is to be hoped that this initiative can definitively clarify this issue. As far as the schedules to perform SCIT are concerned, conventional protocols require a build-up phase followed by a maintenance dose at monthly interval, but short protocols are also available (62). For example, for pollen allergy a short course of four injections of tyrosine-absorbed allergoids enhanced with the adjuvant monophosphoryl lipid A was demonstrated to be effective and safe in children, adolescents and adults with grass and tree pollen allergy (63) and its use is likely to increase in the event of regulatory approval.

\section{Cost-effectiveness of AIT}

The long-term efficacy of AIT after its stopping is the one of the greatest pharmaco-economic advantage of this therapy. In fact, the progressive improvement of symptoms after AIT is paralleled to the decrease in the use of symptomatic drugs. In the first study on SCIT, the analysis of cost-effectiveness was based on the simple calculation of the monetary expen- diture for patients treated with SCIT and symptomatic drugs compared with patients treated only with drugs. Such studies, evaluating mostly SCIT but also SLIT, were reviewed in 2008 by Berto et al., who concluded that AIT, in both routes of administration, may be beneficial to the healthcare systems, because of the better clinical outcome at a cheaper cost or additional benefit at an acceptable extra cost (64). In the ensuing years, the appropriate tools for cost-effectiveness evaluation, such as quality-adjusted life-years (QALYs) and incremental cost effectiveness ratio (ICER) (65) were increasingly used for AIT. In a more recent analysis, 24 studies on health economics of AIT, performed in Northern, Central and Southern Europe and in North America, were reviewed by Hankin and Cox. Nine studies were on SCIT, 10 on SLIT and 5 on both (66). Only one early study comparing the costs for SCIT to costs for drugs in patients with asthma exacerbated by seasonal ragweed exposure failed in detecting a cost-effectiveness, because reduced medication costs were counterbalanced by the costs of immunotherapy (67). However, the study duration was limited to two years of SCIT, thus lacking the post-treatment effect which is now known to enhance the economic advantage of AIT. All the other studies provided, according to Hankin and Cox, "compelling evidence for the cost saving of AIT, whether delivered subcutaneously of sublingually, over symptomatic drug treatment". As far as the comparison between SCIT and SCIT is concerned, most of the analyzed studies reported cost savings favoring SLIT. After this review, two studies comparing SCIT to SLIT were published. Verheggen $e t$ al. used QALYs in a time horizon of 9 years ( 3 years of AIT) to compare the cost-effectiveness of SCIT by a mix of injectable allergoid products to that of SLIT by 5 -grass tablets in patients with grass pollen AR. A cost-utility ratio of the 5 -grass tablet $v$ s. the market mix of injectable allergoids of $€ 12,593$ per QALY in the base case analysis was found. Compared to the allergoid mix the likelihood of the 5-grass tablet to be the most cost-effective treatment was predicted as $76 \%$ at a willingness-to-pay threshold of $€ 20,000$ (68). Different results were reported by Brüggenjürgen et al. in a study involving three nations (Austria, Spain and Switzerland) and comparing SLIT with 5 -grass tablets to a single allergoid and to drug treatment. Both SCIT and SLIT were dominant in the health economic aspects compared to pharmacological symptomatic therapy, while the comparison SCIT-SLIT showed lower total costs of SCIT vs. SLIT for the three nations $(€ 1,368 v$ s. $€ 2,012$, $€ 2,229$ vs. $€ 2,547$, and $€ 1,901$ vs. $€ 2,220$ for Austria, Spain and Switzerland, respectively). Also, higher cost-effectiveness in term of QALYs (SCIT=8.02, SLIT=7.98 QALYs, symptomatic therapy $=7.90)(69)$. In the systematic review by Meadows et al. both SCIT and SLIT were found to be cost-effective when compared with standard treatment from about 6 years (threshold of $£ 20,000-30,000$ per QALY), but the authors 
claimed further research to establish the comparative effectiveness of SCIT compared with SLIT (70).

\section{Differentiation of SCIT and SLIT}

Treatment safety and patients' adherence are the main differences between SCIT and SLIT, as shown by literature.

\section{Safety of SCIT and SLIT}

Safety is the major difference between the two AIT routes. As described above, the reports of fatal reactions to SCIT in the 1980 s $(11,12)$ was a critical issue, which resulted in a limitation of SCIT use and also in the search of alternative routes of administration. SLIT appeared an effective treatment for patients with respiratory allergy with a better safety profile than SCIT, as demonstrated by the absence of fatalities even in the case of consumption of massive dosages. There is a case report of a patient who, after discontinuing SLIT for several months, took all the previously unused doses at once developing an anaphylactic reaction, but surviving (71). Of interest, in the first systematic review on SLIT safety, no difference in the risk of adverse reactions was detected comparing low dose and high dose SLIT products, whereas the dose dependence of SR to SCIT is well known (72). Nevertheless, a risk of anaphylactic reactions in particular circumstances cannot be excluded, as shown by Nolte et al., who analyzed the use of epinephrine in $2408 \mathrm{pa}-$ tients recruited in trials on SLIT tablets with various allergens. A global number of 32 epinephrine administration was necessary (10 for 1-grass pollen, 9 for ragweed pollen, and 13 for dust mite tablets). The reactions treated with epinephrine were not life-threatening. The authors concluded that epinephrine use in SLIT tablets is uncommon, typically occurring in the initial phase of treatment (73). A particular issue concerns the use of protocols directly starting with the maintenance dose, with no build-up, in patients admitted to SLIT because of previous SR to SCIT. In fact, two cases anaphylaxis after the first SLIT dose were described, influencing the subsequent removal of such admission criteria to SLIT (74). Actually, in the most recent US practice parameter update on SLIT it is stated that the first dose of SLIT tablets must be administered in the physician's office and that each patient must be prescribed injectable adrenaline to be used in case of need according to the instructions received (75). This behavior remains mandatory for SCIT, although there was a significant decrease of anaphylactic reactions to SCIT after the identification of uncontrolled asthma in the day of the allergen injection as the major risk factor (13). In a recent EAACI endorsed overview of systematic reviews on AIT, for SCIT about $4 \%$ of systemic reactions were graded as severe (with no fatality), compared with $2 \%$ of such reactions with SLIT (76).

\section{Adherence to SCIT and SLIT}

Adherence to prescribed therapies is a general problem in medicine, as highlighted by Cutler and Everett, who estimated that as many as half of all patients do not adhere to their prescription-medication regimens, with a relevant impact on medical health costs, corresponding to more than $\$ 100$ billion spent each year on avoidable hospitalization (77). The first studies that analyzed adherence to AIT involved patients treated with SCIT. The direct administration of the allergen extract by the physician (or trained nurses) theoretically should ensure good compliance and adherence, but rates of compliance of approximately 50\% were reported instead, both in adults (78) and children (79), the inconvenience from frequents injection and the lack of reimbursement being reported as the major cause of SCIT discontinuation (80). More favorable results were observed using less demanding schedules for injections, with a raise of compliance from $62 \%$ to $88 \%$, provided optimal doses of allergen extracts were administered (81-83). Recent studies substantially confirmed such figures. In a real-life study, the patients who received a SCIT prescription for AR and/or asthma in 2009-2011 were contacted in 2014 and asked about the completion of at least the 3 years of SCIT. A close relationship between allergists and their patients during SCIT and the follow-up period was warranted, resulting in an overall rate of $87.3 \%$ of patients considered adherent (84). In a retrospective chart review of SCIT patients in US between 2003 and 2016, compliance to treatment was evaluated using analysis of variance to compare mean compliance between payer groups. Linear regression showed that age, duration of SCIT therapy and asthma status were not related to the percentage of missed doses, while payer status was statistically predictive of missed doses $(\mathrm{p}=0.02)$. In fact, Medicaid patients missed $34.2 \%$ of doses, followed by Medicare (24.4\%), commercial insurance (19.9\%) and HSN in Massachusetts (18.5\%). The authors concluded that in patients referring to an urban tertiary care setting serving a low-income population, compliance to SCIT was generally high but lower in the Medicaid population (85). As to SLIT, the first studies on compliance and adherence were optimistic, reporting rates of $80-90 \%$ (86). However, in 2010 an investigation based on the prescription data from SLIT products manufacturers revealed that only $13 \%$ was still under treatment after three years, i.e. that duration needed to provide the persistent effect of SLIT over time (87). Given the lack of adherence to SLIT, researchers focused on methods to improve it, demonstrating the importance of patient education and accurate monitoring during the treatment, while the effect of technology-based tools, including online platforms, social media, e-mail, and short message service by phone, is under evaluation (86). In the latest years, studies comparing the compliance and adherence of SCIT and SLIT were published. In a retrospective 
analysis of a community pharmacy database from The Netherlands involving 6486 patients initiating AIT between 1994 and 2009, 2796 patients were treated with SCIT and 3690 were treated with SLIT. Globally, only $18 \%$ of patients $(23 \%$ for SCIT and $7 \%$ for SLIT) completed the required duration of treatment. A premature discontinuation was influenced by the kind of prescriber (a longer persistence was found for patients of general practitioners compared with patients referring to allergists and other medical specialists) by single allergen $v$ s. multiple allergens AIT, by lower socioeconomic status and younger age. The authors claimed for an urgent need to further define the potential barriers for compliance to AIT (87). A study from Spain assessed the effect on compliance of allowing patients to select the route of AIT. Patients were divided into two groups, the study group being formed by patients who chose the route of administration, while control group included patients for whom their physician decided the route. Before starting AIT, all patients received an educative session on the benefits and risks of the treatment, with an additional session in the active group informing about specific characteristics of SCIT and SLIT. After 6 months, 24 of 204 patients in the active group (11\%) and 22 patients of 103 in the control group (21\%) had stopped AIT, this difference being significant $(\mathrm{p}=0.02)$. In the active group no significant difference in compliance was detected between those who preferred SCIT or SLIT, whereas in the control group the number of withdrawals was significantly higher for SLIT than for SCIT $(\mathrm{p}=0.05)$ (88). The rate of compliance was much better than in the Dutch study, but the far shorter observation period (6 months) must be taken into account. A further comparison was done in a retrospective review of 384 patients treated with SCIT or SLIT for at least two years at a tertiary care otolaryngology and allergy practice. SCIT compliance was defined according to injections schedules defined as excellent or good, while for SLIT compliance excellent or good rating was defined according to the number of vials refilled within the expiration date. Excellent or good compliance rates were found in $83.7 \%$ of SCIT patients and in $65.5 \%$ of SLIT patients. Limiting the analysis to excellent compliance rates, a significant difference $(\mathrm{p}>0.05)$ was detected in favor of SCIT (89). The latest study used questionnaires to retrospectively analyze the compliance among 236 patients with AR with or without asthma initiating AIT in 2009 or 2010 . The compliance rates after 3 years were $58.7 \%$ in SCIT treated and $11.6 \%$ in SLIT treated patients. The most common causes of non-compliance were the frequency of injections and the duration of treatment, for SCIT, the inconvenience (to take the allergen extract everyday) and the improvement without treatment for SLIT (91). Globally viewing the results of these studies, it is apparent that, although there is margin of improvement in SCIT compliance, prescribers must be committed to greatly improving SLIT compliance, which is very lacking.

\section{Conclusions}

AIT has unique characteristics among treatments for respiratory allergy, that rely on its ability to induce immunological tolerance to the specific allergen. Currently, two routes of administration are available, namely SCIT and SLIT, that share a number of patterns but differ for others. In particular, a large number of meta-analyses support the efficacy of both routes, without clear evidence of superiority over one another, as acknowledged by recent position papers (92) and analysis by opinion leaders (93). Also, comparable cost-effectiveness was found, especially when the treatment is stopped after adequate duration, moreover the clinical effects persists, with great advantage over symptomatic drug therapy. However, since several NPPs are still present on the market alongside the registered products, a clarification is needed on the existence of different brands and the fact that the indication of an AIT should take into account the quality of the product.

This makes it mandatory to inform prescribers that specific products, rather than AIT, SLIT or SCIT, should be considered as a whole, based on the fact that among the many different products available, for some of them the effectiveness has been demonstrated, while for others it has not.

As far as safety and compliance are concerned, there are differences between SCIT and SLIT. The former has substantially reduced the risk of severe SR following the acknowledgement of the importance of uncontrolled asthma in favoring such reactions, but the safety profile of SCIT is inferior when compared with SLIT. The opposite occurs with compliance, all recent studies reporting rates of compliance much lower for SLIT. This warrants commitment by physicians to improve the outcome, as recently reviewed, by "better patient education at the beginning of treatment, sharing with patients the decision on which type of immunotherapy to select and showing sincere interest in their treatment concerns" (94).

\section{Future perspective}

At the time AIT was introduced (6), all medications were available only in galenic formulation, and in fact AIT was firstly administered through galenic type preparations, obtained directly from the allergenic source, for example grass pollen. In the following decades, the progress in drug development was not mirrored by similar technological improvements in AIT, that, despite an undeniable evolution (94) remained long based on NPP, i.e. allergen extracts prepared for single patients, firstly for SCIT and later also for SLIT, and the quality of these products was uneven. Currently, the need for new products to fulfill the requirements of the regulatory agencies to achieve approval and license to commerce prompted a significant improvement of quality and resulted in major advances, such as the inclusion of SLIT tablets for mite-induced allergic asthma in the GINA 
guidelines for allergic asthma (41). Moreover, according to Nolte and Maloney, the high allergen concentrations administered with SLIT tablets may silence reactive T cells via anergy/ deletion. This process reverses the immune deviation of weakly primed Th2 responses to inhalants towards, typical of allergic subjects, possibly resulting in long term protection against atopic sensitization (93). According to a document from the Agency for Healthcare Research and Quality, the effects of SCIT and SLIT on asthma are not superimposable (95). Actually, the recent EAACI guidelines on AIT for mite-induced asthma gave the recommendations for the different allergen products for AIT. Mite tablets were recommended for adults with controlled or partially controlled asthma, while SCIT was recommended for adults and children and SLIT drops were recommended for children, provided products with demonstration of efficacy by DBPC trials are used and asthma is well controlled (96). Still, in children AIT remains underused and its evidence is challenging due to heterogeneity among studied populations, selection of potential responders, products and outcomes (97), as well as for recognizing optimal schedules of administration (98). The identification of efficacy biomarkers able to predict or monitor the AIT efficacy in early stage is likely to improve the treatment outcome of AIT (99), by avoiding its use in potentially poor responders while supporting the adequate duration in potential responders. In the near future, new advances in AIT are likely, including further methods of administration by intralymphatic or epicutaneous route, which are already supported by several studies, use of recombinant allergens (and hypoallergenic variants), as well as of $\mathrm{T}$ - and B-cell peptide approaches (100). However, the effectiveness of these new approaches still has to be compared with the latest generation of SLIT tablets, which are acknowledged as reference products for AIT.

Finally, regardless the route of administration, high quality trials are warranted to demonstrate the preventive effects of AIT, which was reported in some studies, on the development of new sensitizations and allergic comorbidities with a progressive multiorgan involvement (101).

\section{Executive Summary}

- AIT is the only treatment for respiratory allergy which works on the causes of allergy and not only on symptoms.

- The first method of AIT was SCIT, followed by SLIT in the 1980s. The two routes of administration are now considered of comparable efficacy and produce a similar cost-effectiveness, particularly when the treatment is stopped after appropriate duration.

- Some outcomes show difference between SCIT and SLIT, concerning safety, which is better for SLIT, and treatment compliance, which favors SCIT.
- The new generation of SLIT tablets has achieved a significant advance in quality, that resulted in important accomplishments, such as the inclusion in the GINA guidelines as a therapy for dust-mite induced asthma.

\section{Conflict of interests}

The authors declare that they have no conflict of interests.

\section{References}

1. Cox L, Li JT, Nelson H, Lockey R. Joint Task Force on Practice Parameters; American Academy of Allergy, Asthma and Immunology; American College of Allergy, Asthma and Immunology; Joint Council of Allergy, Asthma and Immunology. Allergen immunotherapy: a practice parameter second update. J Allergy Clin Immunol 2007;120(3 Suppl):S25-85.

2. Akdis CA, Akdis M. Mechanisms of allergen-specific immunotherapy and immune tolerance to allergens. World Allergy Organ J 2015;8(1):17. *The exhaustive explanation of how AIT works.

3. Palomares O, Akdis M, Martín-Fontecha, et al. Mechanisms of immune regulation in allergic diseases: the role of regulatory $\mathrm{T}$ and $\mathrm{B}$ cells. Immunol Rev 2017;278(1):219-236.

4. Głobińska A, Boonpiyathad T, Satitsuksanoa P, et al. Mechanisms of allergen-specific immunotherapy: diverse mechanisms of immune tolerance to allergens. Ann Allergy Asthma Immunol 2018;121(3):306-12.

5. Incorvaia C. One more step towards a deeper understanding of the mechanisms of allergen immunotherapy. EBioMedicine 2018;37:34-35.

6. Cohen SG, Frankland AW, Dworetzky M. Noon and Freeman on prophylactic inoculation against hay fever. J Allergy Clin Immunol 2003;111(5):1142-50.

7. Scadding GK, Brostoff J. Low dose sublingual therapy in patients with allergic rhinitis due to house dust mite. Clin Allergy 1986;16(5):483-91.

8. Johansson SG, Bennich H, Wide L. A new class of immunoglobulin in human serum. Immunology 1968;14(2):265-72. **A major step in understanding the pathophysiology of allergy.

9. Levy DA, Lichtenstein LM, Goldstein EO, Ishizaka K. Immunologic and cellular changes accompanying the therapy of pollen allergy. J Clin Invest 1971;50(2):360-69. * The first recognition of the immunologic modifications induced by AIT.

10. Rocklin RE. Immune mechanisms in allergen-specific immunotherapy. Clin Immunol Immunopathol 1989;53(2 Pt 2):S119-31.

11. [No authors listed]. CSM Update: Desensitising vaccines. Br Med J (Clin Res Ed) 1986;293(6552):948.

12. Lockey RF, Benedict LM, Turkeltaub PC, Bukantz SC. Fatalities from immunotherapy (IT) and skin testing (ST). J Allergy Clin Immunol 1987;79(4):660-77.

13. Epstein TG, Liss GM, Murphy-Berendts K, Bernstein DI. Risk factors for fatal and nonfatal reactions to subcutaneous immunotherapy: National surveillance study on allergen immunotherapy (2008-2013). Ann Allergy Asthma Immunol 2016;116(4):35459. ${ }^{*}$ The study that highlighted the risk factors for severe reactions to SCIT.

14. Canonica GW, Passalacqua G. Noninjection routes for immunotherapy. J Allergy Clin Immunol 2003;111:437-48. 
15. Larsen JN, Broge I, Jacobi H. Allergy immunotherapy: the future of allergy treatment. Drug Discov Today 2016;21(1):26-37.

16. Walker E, Hernandez AV, Kattan MW. Meta-analysis: Its strengths and limitations. Cleve Clin J Med 2008;75(6):43139.

17. Enkin M, Hetherington J. Collecting the evidence systematically. Ensuring that it is complete and up-to-date. Int J Technol Assess Health Care 1996;12(2):276-79.

18. Abramson MJ, Puy RM, Weiner JM. Is allergen immunotherapy effective in asthma? A meta-analysis of randomized controlled trials. Am J Respir Crit Care Med 1995;151:969-74.

19. Abramson MJ, Puy RM, Weiner JM. Allergen immunotherapy for asthma. Cochrane Database Syst Rev 2003;4:CD00118.

20. Calderon MA, Alves B, Jacobson M, et al. Allergen injection immunotherapy for seasonal allergic rhinitis. Cochrane Database Syst Rev 2007;1:CD001936.

21. Abramson MJ, Puy RM, Weiner JM. Injection immunotherapy for asthma. Cochrane Database Syst Rev 2010;8:CD001186. **The systematic review and meta-analysis, including the largest number of trials, focusing the efficacy and safety of SCIT.

22. Matricardi PM, Kuna P, Panetta V, et al. Subcutaneous immunotherapy and pharmacotherapy in seasonal allergic rhinitis: a comparison based on meta-analyses. J Allergy Clin Immunol 2011;128(4):791-99.

23. Lu Y, Xu L, Xia M, et al. The efficacy and safety of subcutaneous immunotherapy in mite-sensitized subjects with asthma: a meta-analysis. Respir Care 2015;60(2):269-78.

24. Wilson DR, Torres-Lima M, Durham S. Sublingual immunotherapy for allergic rhinitis: systematic review and meta-analysis. Allergy 2005;60:4-12. *The first meta-analysis on the efficacy of SLIT, showing its feasibility as an option to SCIT.

25. Penagos M, Compalati E, Tarantini F, et al. Efficacy of sublingual immunotherapy in the treatment of allergic rhinitis in pediatric patients 3-18 years of age: a meta analysis of randomized placebo-controlled, double blind trials. Ann Allergy Asthma Immunol 2006;97:141-48.

26. Calamita Z, Saconato H, Pela AB, Atallah AN. Efficacy of sublingual immunotherapy in asthma: systematic review of randomized-clinical trials using the Cochrane Collaboration method. Allergy 2006;61:1162-72.

27. Penagos M, Passalacqua G, Compalati E, et al. Meta-analysis of the efficacy of sublingual immunotherapy in the treatment of allergic asthma in pediatric patients, 3 to 18 years of age. Chest 2008;133:599-609.

28. Compalati E, Passalacqua G, Bonini M, Canonica GW. The efficacy of sublingual immunotherapy for house dust mites respiratory allergy: results of a GA2LEN meta-analysis. Allergy 2009;64:1570-79.

29. Di Bona D, Plaia A, Scafidi V, et al. Efficacy of sublingual immunotherapy with grass allergens for seasonal allergic rhinitis: a systematic review and meta-analysis. J Allergy Clin Immunol 2010;126:558-66.

30. Radulovic S, Calderon MA, Wilson D, Durham S. Sublingual immunotherapy for allergic rhinitis. Cochrane Database Syst Rev 2010;12:CD002893.

31. Normansell R, Kew KM, Bridgman AL. Sublingual immunotherapy for asthma. Cochrane Database Syst Rev 2015;(8):CD011293.

32. Dhami S, Nurmatov U, Arasi S, et al. Allergen immunotherapy for allergic rhinoconjunctivitis: A systematic review and meta-analysis. Allergy 2017;72(11):1597-631.
33. Dhami S, Kakourou A, Asamoah F, et al. Allergen immunotherapy for allergic asthma: A systematic review and meta-analysis. Allergy 2017;72(12):1825-48.

34. Huang Y, Wang C, Wang X, et al. Efficacy and safety of subcutaneous immunotherapy with house dust mite for allergic rhinitis: A meta-analysis of randomized controlled trials. Allergy 2019;74(1):189-92.

35. Muraro A, Roberts G, Halken S, et al. EAACI guidelines on allergen immunotherapy: Executive statement. Allergy 2018;73(4):739-43.

36. Durham SR, Yang WH, Pedersen MR, et al. Sublingual immunotherapy with once daily grass allergen tablet: a randomized controlled trial in seasonal allergic rhinoconjunctivitis. J Allergy Clin Immunol 2006;117:802-09. *The first study on the efficacy of the new generation of tablets for SLIT.

37. Didier A, Malling HJ, Worm M, et al. Optimal dose, efficacy, and safety of once daily sublingual immunotherapy with a 5-grass pollen tablet for seasonal allergic rhinitis. J Allergy Clin Immunol 2007;120:1338-45.

38. Wahn U, Tabar A, Kuna P, et al. SLIT Study Group. Efficacy and safety of 5-grass pollen sublingual immunotherapy tablets in pediatric allergic rhinoconjunctivitis. J Allergy Clin Immunol 2009; 123:160-66.

39. Devillier P, Brehler R, Sastre J, et al. The clinical development of specific immunotherapies: specific methodological issues and clinical interpretation of results. Allergy 2010;65(suppl 92):567.

40. Mosbech H, Deckelmann R, de Blay F, et al. Standardized quality (SQ) house dust mite sublingual immunotherapy tablet (ALK) reduces inhaled corticosteroid use while maintaining asthma control: a randomized, double-blind, placebo-controlled trial. J Allergy Clin Immunol 2014;134(3):568-75. *The randomized trial showing the efficacy of dust mite SLIT tablets which resulted in inclusion of AIT for asthma treatment in the GINA guideline.

41. Global Strategy for Asthma Management and Prevention (2017 update) www.ginasthma.org (accessed January 8, 2018).

42. Bergmann KC, Demoly P, Worm M, et al. Efficacy and safety of sublingual tablets of house dust mite allergen extracts in adults with allergic rhinitis. J Allergy Clin Immunol 2014;133(6):1608-14.

43. Marogna M, Spadolini I, Massolo A, et al. Randomized controlled open study of sublingual immunotherapy for respiratory allergy in real-life: clinical efficacy and more. Allergy 2004;59(11):1205-10.

44. Incorvaia C, Barberi S, Pastorello E, et al. The growing importance of real-life studies in allergen immunotherapy. Eur Ann Allergy Clin Immunol 2019;51(3):115-21.

45. Zielen S, Devillier P, Heinrich J, Richter H, Wahn U. Sublingual immunotherapy provides long-term relief in allergic rhinitis and reduces the risk of asthma: a retrospective, real-world database analysis. Allergy 2018;73(1):165-177. *The large study demonstrating the utility of real-live studies on AIT.

46. Wahn U, Bachert C, Heinrich J, Richter H, Zielen S. Real-world benefits of allergen immunotherapy for birch pollen-associated allergic rhinitis and asthma. Allergy 2019;74(3):594-604.

47. Devillier P, Molimard M, Ansolabehere X, et al. Immunotherapy with grass pollen tablets reduces medication dispensing for allergic rhinitis and asthma: a retrospective database study in France. Allergy 2019;74(7):1317-26.

48. Agache I, Sugita K, Morita H, et al. The Complex Type 2 Endotype in Allergy and Asthma: From Laboratory to Bedside. Curr Allergy Asthma Rep 2015;15(6):29.

49. Boonpiyathad T, Sözener ZC, Satitsuksanoa P, et al. Immunologic mechanisms in asthma. Semin Immunol 2019;46:101333. 
50. Şahin E, Bafaqeeh SA, Güven SG, et al. Mechanism of action of allergen immunotherapy. Am J Rhinol Allergy 2016;30(5):1-3.

51. Passalacqua G. Specific immunotherapy: beyond the clinical scores. Ann Allergy Asthma Immunol 2011;107(5):401-06.

52. Penagos M, Eifan AO, Durham SR, et al. Duration of allergen immunotherapy for long-term efficacy in allergic rhinoconjunctivitis. Curr Treat Options Allergy 2018;5(3):275-90.

53. Jacobsen L, Niggemann B, Dreborg S, et al. Specific immunotherapy has long-term preventive effect of seasonal and perennial asthma: 10-year follow-up of the PAT study. Allergy 2007;62:943-48.

54. Rudman Spergel AK, Minnicozzi M, Wheatley LM, et al. Is allergen immunotherapy in children disease modifying? A Review of the evidence. Curr Allergy Asthma Rep 2018;18(9):47.

55. Des Roches A, Paradis L, Menardo JL, et al. Immunotherapy with a standardized Dermatophagoides pteronyssinus extract. VI. Specific immunotherapy prevents the onset of new sensitizations in children. J Allergy Clin Immunol 1997;99(4):450-53.

56. Martignago I, Incorvaia C, Ridolo E. Preventive actions of allergen immunotherapy: the facts and the effects in search of evidence. Clin Mol Allergy 2017; 15:13.

57. Di Bona D, Plaia A, Leto-Barone MS, et al. Efficacy of allergen immunotherapy in reducing the likelihood of developing new allergensensitizations: a systematic review. Allergy 2017;72(5):691-704.

58. Cox L, Jacobsen L. Comparison of allergen immunotherapy practice patterns in the United States and Europe. Ann Allergy Asthma Immunol 2009; 103:451-59.

59. 59. Nelson HS. Multiallergen immunotherapy for allergic rhinitis and asthma. J Allergy Clin Immunol 2009;123:763-69.

60. Calderon MA, Cox L, Casale T, et al. Multiple-allergen and single-allergen immunotherapy strategies in polysensitized patients: looking at the published evidence. J Allergy Clin Immunol 2012;129:929-34.

61. Wheatley LM, Wood R, Nadeau K, et al. Mind the gaps: Clinical trial concepts to address unanswered questions in aeroallergen immunotherapy-An NIAID/AHRQ Workshop. J Allergy Clin Immunol 2019;143:1711-26.

62. Larenas-Linnemann D. Allergen immunotherapy: an update on protocols of administration. Curr Opin Allergy Clin Immunol 2015;15(6):556-67.

63. Rosewich M, Lee D, Zielen S. Pollinex Quattro: an innovative four injections immunotherapy in allergic rhinitis. Hum Vaccin Immunother 2013;9(7):1523-31.

64. Berto P, Frati F, Incorvaia C. Economic studies of immunotherapy: a review. Curr Opin Allergy Clin Immunol 2008;8(6):585-89.

65. Taylor C, Jan S. Economic evaluation of medicines. Aust Prescr 2017;40(2):76-78.

66. Cox L. Allergy immunotherapy: what is the evidence for cost saving? Curr Opin Allergy Clin Immunol 2014;14(4):363-70.

67. Creticos PS, Reed CE, Norman PS, et al. Ragweed immunotherapy in adult asthma. N Engl J Med 1996;334(8):501-06.

68. Verheggen BG, Westerhout KY, Schreder CH, et al. Health economic comparison of SLIT allergen and SCIT allergoid immunotherapy in patients with seasonal grass-allergic rhinoconjunctivitis in Germany. Clin Transl Allergy 2015;5:1.

69. Brüggenjürgen B, Reinhold T. Cost-effectiveness of grass pollen subcutaneous immunotherapy (SCIT) compared to sublingual immunotherapy (SLIT) and symptomatic treatment in Austria, Spain, and Switzerland. J Med Econ 2018;21(4):374-81.

70. Meadows A, Kaambwa B, Novielli N, et al. A systematic review and economic evaluation of subcutaneous and sublingual allergen immunotherapy in adults and children with seasonal allergic rhinitis. Health Technol Assess 2013;17(27):vi, xi-xiv, 1-322.

71. Blazowski L. Anaphylactic shock because of sublingual immunotherapy overdose during third year of maintenance dose. Allergy 2008;63(3):374.

72. Gidaro GB, Marcucci F, Sensi L, et al. The safety of sublingual-swallow immunotherapy: an analysis of published studies. Clin Exp Allergy 2005;35:565-71. *The study demonstrating that, differently from SCIT, safety of SLIT is not dose-depending.

73. Nolte H, Casale TB, Lockey RF, et al. Epinephrine use in clinical trials of sublingual immunotherapy tablets. J Allergy Clin Immunol Pract 2017;5(1):84-89.

74. de Groot H, Bijl A. Anaphylactic reaction after the first dose of sublingual immunotherapy with grass pollen tablet. Allergy 2009;64(6):963-64.

75. Greenhawt M, Oppenheimer J, Nelson M, et al. Sublingual immunotherapy: A focused allergen immunotherapy practice parameter update. Ann Allergy Asthma Immunol 2017;118(3):276-82.

76. Nurmatov U, Dhami S, Arasi S, et al. Allergen immunotherapy for allergic rhinoconjunctivitis: a systematic overview of systematic reviews. Clin Transl Allergy 2017;7:24.

77. Cutler DM, Everett W. Thinking outside the pillbox - Medication adherence as a priority for health care reform. N Engl J Med 2010;362:1553-55.

78. Cohn JR, Pizzi A. Determinants of patient compliance with allergen immunotherapy. J Allergy Clin Immunol 1993;91:73477.

79. Lower T, Henry J, Mandik L, et al. Compliance with allergen immunotherapy. Ann Allergy Asthma Immunol 1993;70:480-82.

80. Cox LS, Hankin C, Lockey R. Allergy immunotherapy adherence and delivery route: location does not matter. J Allergy Clin Immunol Pract 2014;2(2):156-60.

81. Tinkelman D, Smith F, Cole WQ, et al. Compliance with an allergen immunotherapy regime. Ann Allergy Asthma Immunol 1995;74:241-46.

82. Ruiz FJ, Jimenez A, Cocoletsi J, et al. Compliance with and abandonment of immunotherapy. Rev Alerg Mex 1997;44:42-44.

83. Rhodes BJ. Patients dropouts before completion of optimal dose, multiple allergen immunotherapy. Ann Allergy Asthma Immunol 1999;82:281-86.

84. Gelincik A, Demir S, Olgaç M, et al. High adherence to subcutaneous immunotherapy in a real-life study from a large tertiary medical center. Allergy Asthma Proc 2017;38(6):78-84.

85. Keefe KR, Ngo-Howard M, Platt MP, et al. Compliance with subcutaneous immunotherapy appointments in an urban tertiary care setting. Am J Rhinol Allergy 2018;32(6):473-77.

86. Incorvaia C, Mauro M, Leo G, et al. Adherence to Sublingual Immunotherapy. Curr Allergy Asthma Rep 2016;16(2):12.

87. Senna G, Lombardi C, Canonica GW, et al. How adherent to sublingual immunotherapy prescriptions are patients? The manufacturers' viewpoint. J Allergy Clin Immunol 2010;126(3):668-69. *The study based on data from producers showing that the long-term adherence to SLIT is low.

88. Kiel MA, Röder E, Gerth van Wijk R, et al. Real-life compliance and persistence among users of subcutaneous and sublingual allergen immunotherapy. J Allergy Clin Immunol 2013;132(2):353-60.

89. Sánchez J. Adherence to allergen immunotherapy improves when patients choose the route of administration: Subcutaneous or sublingual. Allergol Immunopathol (Madr) 2015;43(5):436-41. 
90. Leader BA, Rotella M, Stillman L, et al. Immunotherapy compliance: comparison of subcutaneous versus sublingual immunotherapy. Int Forum Allergy Rhinol 2016;6(5):460-64.

91. Musa F, Al-Ahmad M, Arifhodzic N, et al. Compliance with allergen immunotherapy and factors affecting compliance among patients with respiratory allergies. Hum Vaccin Immunother 2017;13(3):514-17.

92. Roberts G, Pfaar O, Akdis CA, et al. Immunotherapy coming of age: notable advances during the first hundred years. Expert Rev Clin Immunol 2017;13(5):389-92.

93. Pitsios C, Dietis N. Ways to increase adherence to allergen immunotherapy. Curr Med Res Opin 2019;35(6):1027-1031.

94. Nolte H, Maloney J. The global development and clinical efficacy of sublingual tablet immunotherapy for allergic diseases. Allergol Int 2018;67(3):301-08.

95. Lin SY, Azar A, Suarez-Cuervo C, et al. The role of immunotherapy in the treatment of asthma. Rockville, MD: Agency for Healthcare Research and Quality (US) 2018;17(18):EHC029-EF.

Papers of special note have been highlighted as either of interest (*) or of considerable interest $\left(^{* *}\right)$ to readers.
96. Agache I, Lau S, Akdis CA, et al. EAACI guidelines on allergen immunotherapy: house dust mite-driven allergic asthma. Allergy 2019;74(5):855-73.

97. Caffarelli C, Cangemi J, Mastrorilli C, Giannetti A, Ricci G. Allergen-specific Immunotherapy for Inhalant Allergens in Children. Curr Pediatr Rev 2020;16(2):129-139.

98. Kopp MV, König IR, Friedrichs F, et al. Comparison of six different allergen extracts for subcutaneous specific immunotherapy in children: an open-labelled, prospective, controlled observational trial. Int Arch Allergy Immunol 2019;180(4):284-90.

99. Wang W, Yin J. Is it worthy to take full-course immunotherapy for allergic rhinitis? About efficacy biomarker of allergen immunotherapy. Scand J Immunol 2020;91(1):e12817.

100. Gunawardana NC, Durham SR. New approaches to allergen immunotherapy. Ann Allergy Asthma Immunol 2018;121(3):293-05.

101. Halken S, Larenas-Linnemann D, Roberts G, et al. EAACI guidelines on allergen immunotherapy: Prevention of allergy. Pediatr Allergy Immunol 2017;28(8):728-45. 